\section{Creutzfeldt-Jakob Disease: Recommendations for Disinfection and Sterilization}

Prion diseases constitute a unique infection control problem, because prions exhibit unusual resistance to conventional chemical and physical decontamination methods. Recommendations to prevent crosstransmission of infection from medical devices contaminated by Creutzfeldt-Jakob disease (CJD) have been based primarily on prion inactivation studies, and these recommendations cannot be validly extrapolated to modern reprocessing procedures because instrument cleaning is ignored. In recent years, some countries and institutions have made recommendations for processing instruments that are exposed to patients with CJD that are unnecessarily stringent. This is due primarily to the epidemic of bovine spongiform encephalopathy (BSE) in the United Kingdom, which began in 1986, has affected nearly 200,000 cattle, is waning to a conclusion, but in its wake is left an outbreak of human Creutzfeldt-Jakob disease (vCJD), most probably resulting from the consumption of beef products contaminated by central nervous system tissue.

Drs. William Rutala and David Weber from the University of North Carolina School of Medicine have published recommendations that are evidence-based, reasonable, and in direct contrast to some of the more fear-inspired guidelines on the same subject. The authors consider inactivation data but also use epidemiological studies of prion transmission, infectivity of human tissues, and efficacy of removing microorganisms by cleaning. On the basis of the scientific data, only critical (eg, surgical instruments) and semicritical devices contaminated with high-risk tissue (ie, brain, spinal cord, and eye tissue) from high-risk patients (those with known or suspected infection) require special treatment.
Some the major points on processing strategies are as follows:

1. For high-risk tissues (brain, spinal cord, eye), highrisk patients, or critical or semicritical devices:

a. Devices that are readily cleaned: Clean, then steritize by steam sterilization at $121^{\circ} \mathrm{C}$ to $132^{\circ} \mathrm{C}$ for 60 minutes or prevacuum steam sterilization at $134^{\circ} \mathrm{C}$ for 18 minutes.

b. Devices that are difficult or impossible to clean: Discard, or process as follows: place in a liquid such as water or saline, to limit adherence of material, then autoclave as described above or soak in 1 $\mathrm{N}$ sodium hydroxide for 1 hour. Follow with conventional cleaning, wrapping, and sterilization.

2. For low-risk tissues (cerebrospinal fluid, kidney, liver, spleen, lung, lymph nodes), high-risk patients, or critical or semicritical devices:

a. Clean the device, and then use conventional protocols for heat or chemical sterilization or high-level disinfection.

b. Use standard disinfection to clean environmental surfaces.

3. For no-risk tissues, high-risk patients, critical or semicritical devices:

a. Same recommendations as for low-risk tissues. This section notes that endoscopes, other than neurosurgical endoscopes, would be contaminated only with no-risk material, and therefore standard cleaning and high-level disinfection would be adequate.

The article also lists general infection control precautions on such matters as handling of tissue samples, medical waste, and laundry, and it provides suggestions for dealing with possible occupational exposures.

FROM: Rutala WA, Weber DJ. Creutzfeldt-Jakob disease: recommendations for disinfection and sterilization. Clin Infect Dis 2001;32:1348-1356. 